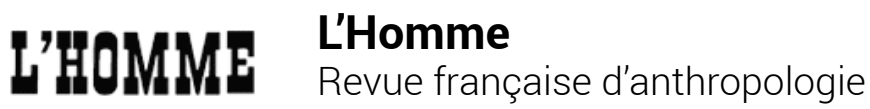

175-176 | juillet-septembre 2005

Vérités de la fiction

\section{Fiction, folie, fétichisme}

Claude Lévi-Strauss entre Comte et La Comédie humaine

Frédéric Keck

\section{(2) OpenEdition}

Journals

Édition électronique

URL : http://journals.openedition.org/lhomme/29540

DOI : $10.4000 /$ Ihomme. 29540

ISSN : 1953-8103

Éditeur

Éditions de l'EHESS

Édition imprimée

Date de publication : 15 octobre 2005

Pagination : 203-218

ISBN : 2-7132-2035-1

ISSN : 0439-4216

Référence électronique

Frédéric Keck, «Fiction, folie, fétichisme », L'Homme [En ligne], 175-176 | juillet-septembre 2005, mis en ligne le 01 janvier 2007, consulté le 19 avril 2019. URL : http://journals.openedition.org/

Ihomme/29540; DOI : 10.4000/lhomme.29540 
chercher : repérer : avancer

Cet article est disponible en ligne à l'adresse :

http://www.cairn.info/article.php?ID REVUE=LHOM\&ID NUMPUBLIE=LHOM 175\&ID ARTICLE=LHOM 1750203

Fiction, folie, fétichisme. Claude Lévi-Strauss entre Comte et La Comédie humaine

par Frédéric KECK

| Éditions de l'EHESS | L'Homme

$2005 / 3-4$ - № $175-176$

ISSN 0439-4216 | ISBN 2-7132-2035-1 | pages 203 à 218

Pour citer cet article :

-Keck F., Fiction, folie, fétichisme. Claude Lévi-Strauss entre Comte et La Comédie humaine, L'Homme 2005/ 3-4, $\mathrm{N}^{\circ}$ 175-176, p. 203-218.

Distribution électronique Cairn pour les Éditions de l'EHESS.

(C) Éditions de l'EHESS. Tous droits réservés pour tous pays.

La reproduction ou représentation de cet article, notamment par photocopie, n'est autorisée que dans les limites des conditions générales d'utilisation du site ou, le cas échéant, des conditions générales de la licence souscrite par votre établissement. Toute autre reproduction ou représentation, en tout ou partie, sous quelque forme et de quelque manière que ce soit, est interdite sauf accord préalable et écrit de l'éditeur, en dehors des cas prévus par la législation en vigueur en France. Il est précisé que son stockage dans une base de données est également interdit. 


\title{
Fiction, folie, fétichisme
}

\section{Claude Lévi-Strauss entre Comte et La Comédie humaine}

\author{
Frédéric Keck
}

LE CONCEPT de fétichisme a été exclu du champ anthropologique par Marcel Mauss parce qu'il reposait sur un «immense malentendu » quant au culte des objets dans les sociétés d'Afrique noire ${ }^{1}$. Pourtant, la réflexion sur le fétichisme ne désignait pas seulement une religion observable dans certaines sociétés supposées primitives, mais plus profondément un certain état de la pensée humaine, présent dans toutes les sociétés à tout moment du temps historique: ce qu'Auguste Comte appelait «l'état théologique ou fictif $»^{2}$. Si le terme de fétichisme dérive du portugais feitiço et du latin facticius, il désigne aussi bien le «factice » - l'erreur, l'imposture, l'ensorcellement, le charlatanisme - que le «fictif »- l'imaginaire, le fabriqué, le féerique, le créé. L'ambivalence du rapport du fondateur du positivisme au concept de fétichisme serait alors l'indice d'une ambivalence du savoir

1. Voir Alfonso Iacono (1992: 116). Le concept de fétichisme a été introduit dans la littérature anthropologique en 1760 par Charles de Brosses dans $D u$ culte des dieux fétiches, et rejeté à la fin du XIX ${ }^{\mathrm{e}}$ siècle par Max Müller en 1889 dans Natural Religions, puis par Marcel Mauss en 1907 dans L'Année sociologique.

2. Voir Comte (1998 : 21). Georges Canguilhem (1994: 81) a montré que le concept de fétichisme ne désigne pas chez Comte un stade historique de la pensée, mais une structure de signification dans le rapport de l'homme au monde: «Sous le nom de fétichisme, Auguste Comte a cherché à construire une théorie abstraite et totale des rapports de la religion et de la nature humaine. [...] La théorie comtienne des origines de la forme religieuse de penser repose moins sur la connaissance descriptive de formes sociales chronologiquement initiales que sur l'éclaircissement de la signification permanente d'une réaction de l'homme à sa situation originaire ». Jean Pouillon (1975: 116) prolonge cette hypothèse en montrant que le concept de fétichisme désigne ce que Lévi-Strauss appelle une limite de la pensée symbolique, qui permettrait de faire une carte des usages des fétiches plutôt qu'une philosophie de l'histoire des religions: "S'il en est bien ainsi, et si l'on veut continuer à parler de fétiche, il faut replacer celui-ci dans le champ de la pensée symbolique, et se demander non plus ce qu'est le fétichisme, mais quand et à propos de quoi on parle de fétiche ».

Cet article a d'abord fait l'objet d'un exposé au séminaire de Jean-Paul Colleyn \& Jean Jamin sur l'anthropologie de la fiction (Ehess, 3 mars 2005). Je les remercie, ainsi que Vincent Debaene, pour leurs remarques. 
anthropologique qu'il met en place - avec son schéma d'un progrès nécessaire des « religions primitives » aux «sciences modernes »- quant au statut de la fiction. Les commentateurs de Comte se sont étonnés que le fétichisme, d'abord considéré comme le premier stade de l'état théologique, soit un thème de plus en plus obsédant dans l'œuvre politique de Comte, au point de devenir un modèle pour la constitution d'une "religion positiviste », parfois qualifiée de "néo-fétichisme » ${ }^{3}$. Certains ont attribué à la folie de Comte un tel passage de la philosophie des sciences à l'organisation d'une nouvelle religion ; mais c'est ignorer le rapport profond chez Comte entre positivisme et fétichisme, et plus généralement entre le savoir anthropologique et un certain exercice de la fiction, qui confine avec la folie d'une façon qu'il faut tenter d'explorer ${ }^{4}$. L'ambivalence de Comte quant au fétichisme serait alors l'indice d'une ambivalence du savoir anthropologique, qui ne dénonce l'usage irrationnel de la fiction que pour le réintroduire par la bande au cœur même des opérations rationnelles ${ }^{5}$. La question que pose le fétichisme est en effet celle du type de vérité auquel peut prétendre un discours anthropologique mettant les faits en relation sans passer par une expérience subjective. Comment un savoir total sur l'homme est-il possible qui ne passe pas par l'expérience synthétisante d'un sujet ? La fiction vient répondre à cette question constitutive du savoir moderne et de ses ambivalences. Alors, peut-être, Mauss a-t-il voulu exorciser, en bannissant le fétichisme, non pas seulement une erreur théorique sur les « religions primitives", mais une tentation, voire une nécessité, du discours anthropologique : celle de recourir au mode littéraire de la fiction pour exposer dans sa vérité subjective l'objet de l'enquête ethnographique ${ }^{6}$. C'est l'hypothèse que nous suivrons dans cette étude sur les rapports entre Lévi-Strauss, Comte et Balzac.

3. Voir Littré (1862). Littré a diffusé dans la pensée républicaine l'idée selon laquelle il faudrait lire la philosophie des sciences de Comte indépendamment de sa philosophie religieuse et politique, construite selon lui dans sa "seconde carrière » sous le coup de la folie. Contre cette thèse, et pour une défense de l'unité de la pensée de Comte, voir Lévy-Bruhl (1900), Delvolvé (1932), Gouhier (1987). Jean Delvolvé (1932 : 182) est le premier à relire toute la pensée de Comte à partir du thème du fétichisme (dont Lévy-Bruhl ne parle étrangement pas), en affirmant que le «néofétichisme » de Comte doit être compris dans le cadre d'une réflexion métaphysique et morale sur la place de l'expérience subjective dans le cadre social organisé par des lois : "Comte a jugé nécessaire de rapporter simultanément la synthèse philosophique des sciences et la construction des cadres idéaux de la pratique humaine, non pas aux lois abstraites de la sociologie, mais à la nature concrète de l'existence humaine, complexe total, inaccessible en fait à l'analyse scientifique proprement dite, donnant lieu à la création d'une science suprême, concrète, de caractère unique : la morale. Il a affirmé que cette nature concrète est pratiquement saisissable de façon immédiate dans le sentiment, et il a conçu sous la forme d'une religion le développement institutionnel de la synthèse subjective, ainsi caractérisée comme construction idéale inséparablement théorique et pratique ». Delvolvé exclut cependant la dimension fictive du fétichisme, qu'il considère peut-être comme immorale : "[Comte] n'a pas compris que ce qu'il appelle fictions valait comme intuition obscure et expression imparfaite de vérités profondes » (Ibid. : 239).

4. Je poserai ici seulement la question de l'usage scientifique de la fiction chez Comte. Reste ouverte la question de son usage politique dans le positivisme comtien, notamment à partir du statut énigmatique des «organismes fictifs» (Descombes 1978).

5. Voir Latour (1996), Schaeffer (1999).

6. Comte a en effet cessé toute lecture scientifique après sa rencontre avec Clotilde de Vaux, pour ne plus lire que de la littérature et écouter de l'opéra (voir Lepenies 1990). 
Dans un texte situé à l'exact milieu de La Pensée sauvage, Claude Lévi-Strauss insère une référence énigmatique à l'auteur de La Comédie humaine. Il vient de dissoudre "l'illusion totémique » selon laquelle il existerait dans certaines sociétés un système totémique fait d'interdits alimentaires et de prescriptions matrimoniales, et a mis en lumières, sur les décombres de cette institution imaginaire, une forme de pensée observable en droit dans toutes les sociétés, qui se constitue au moyen de schèmes reliant des espèces naturelles et des groupes sociaux. Est ainsi découvert un champ d'études entièrement neuf, celui de la «science du concret », qui doit montrer les opérations logiques à travers lesquelles l'esprit humain classe les espèces naturelles pour classer les individus socialisés. Mais aussitôt après avoir ouvert ce programme - que toute l'anthropologie structurale doit remplir - Lévi-Strauss (1962 : 159) ajoute une clause de prudence :

«Nous ne voulons pas dire que la vie sociale, les rapports entre l'homme et la nature, sont une projection, sinon même un résultat, d'un jeu conceptuel qui se déroulerait dans l'esprit. "Les idées", écrivait Balzac, "sont en nous un système complet, semblable à l'un des règnes de la nature, une sorte de floraison dont l'iconographie sera retracée par un homme de génie qui passera pour fou peut-être". Mais à qui tenterait l'entreprise, il faudrait sans doute plus de folie que de génie. ${ }^{7}$

Suit alors un texte célèbre dans lequel Lévi-Strauss (Ibid. : 160) s'oppose au marxisme de Sartre, en affirmant que le but des sciences humaines n'est pas de reconstituer la praxis des individus, totalité infinie formée par la dialectique des actions humaines, mais l'ensemble fini des schèmes à travers lesquels les individus pensent leurs pratiques, en vertu du fait que:

«entre praxis et pratiques s'intercale toujours un médiateur, qui est le schème conceptuel par l'opération duquel une matière et une forme, dépourvues l'une et l'autre d'existence indépendante, s'accomplissent comme structures, c'est-à-dire comme êtres à la fois empiriques et intelligibles. C'est à cette théorie des superstructures, à peine esquissée par Marx que nous souhaitons contribuer [...] parce que l'ethnologie est d'abord une psychologie. »

Il est remarquable que la référence à Balzac et à la taxinomie précède la discussion avec Sartre et Marx sur la praxis. Il faut en effet replacer La Pensée sauvage dans le contexte d'une rivalité avec Sartre, dont Lévi-Strauss a lu attentivement la Critique de la raison dialectique, sur le sens philosophique à donner au marxisme et aux sciences humaines(Keck 2004). Tout se passe alors comme si la référence à Balzac permettait de conjurer par avance la tentation d'un «homme total », qui fut constitutive de l'alliance entre le marxisme et les sciences humaines en France ${ }^{8}$. Sartre cherche en effet à retrouver dans la praxis d'un individu - Genet, Baudelaire, Flaubert - la totalité des déterminations qui pèsent sur

7. La citation de Balzac est tirée de Louis Lambert.

8. Voir Bruno Karsenti (1997). Le lien entre le réalisme balzacien et le thème marxiste de «l'homme total » a été noué par Georg Lukács (1967: 10) : "C'est seulement lorsque l'homme total s'offre à nous comme la tâche sociale historique incombant à l'humanité, c'est seulement lorsque nous considérons la profession artistique comme la représentation des principaux tournants de ce processus avec la richesse des éléments qui y sont agissants, c'est seulement ... /... 
lui et qu'il parvient à nier par l'écriture, véritable acte de liberté qui pose l'ordre culturel du langage dans l'ordre «practico-inerte » de la nature, et qui se retrouve constamment hanté par cette nature qu'il n'a jamais suffisamment niée, en sorte que l'individu figure au titre de «totalité intotalisable » ou comme «mouvement infini de totalisation » (Sartre 1960 : 187). En citant Balzac, Lévi-Strauss indique avec ironie qu'un tel projet de totalisation, s'il devient projet pour une conscience, est impossible ; mais s'il anime inconsciemment toutes les opérations de la pensée sauvage, il peut constituer l'horizon de l'enquête anthropologique.

Le rêve taxinomique de Balzac figure ainsi chez Lévi-Strauss comme alternative au projet totalisateur de Sartre. S'il est possible que toutes les classifications sociales et naturelles figurent dans l'espace d'un même tableau, celui qui voudrait peindre ce tableau serait plus proche de la folie que du génie. C'est pour quoi il faut en passer par les "Sauvages », et toutes les formes de pensée et de pratiques marginales et dénigrées, pour comprendre le véritable mouvement de totalisation, inaccessible au savant livré à l'exigence de séparer les faits: c'est que dans ces formes de pensée inconscientes d'elles-mêmes, les faits sont pensés ensemble d'un même mouvement sans pour autant passer par une conscience. «Il n'y a rien au monde que les Sauvages, les paysans et les gens de province pour étudier à fond leurs affaires dans tous les sens ; aussi quand ils arrivent de la Pensée au Fait, trouvez-vous les choses complètes. » Cette citation de Balzac, extraite du Cabinet des Antiques, mise en épigraphe dans La Pensée sauvage, donne tout le sens de l'ouvrage. Il s'agit pour Lévi-Strauss d'intégrer dans la pensée scientifique, caractérisée par l'exigence de clarté et de distinction, une "pensée sauvage » qui a déjà analysé tout le réel mais de façon déraisonnablement précipitée. C'est pourquoi seule la rencontre en elle-même improbable entre la pensée scientifique et la pensée sauvage - favorisée selon Lévi-Strauss (1962: 321) par la révolution des sciences de l'information et de la communication: physique quantique, génétique, cybernétique - donne au "procès tout entier de la connaissance humaine [... ] le caractère d'un système clos ».

Il y a donc bien une tentation totalisatrice dans la pensée scientifique de LéviStrauss, renforcée par sa confrontation avec la méthode de totalisation qu'est la dialectique philosophique sartrienne ; mais elle est transférée sur une autre scène par la référence au projet littéraire de Balzac. Cette tentation est classiquement décrite comme celle de l'introspection psychologique. Dire que «l'ethnologie est une psychologie », c'est certes montrer que les classifications sociales et naturelles des sociétés sauvages doivent être étudiées comme des opérations mentales ou des schèmes conceptuels, mais c'est aussi courir le risque de projeter sur elles «un jeu conceptuel qui se déroulerait dans l'esprit ». Dan Sperber (1974: 69) a ainsi reproché à

[suite de la note 8] lorsque l'esthétique de l'art fixe comme mission d'indiquer la voie de l'humanité, que le contenu de la vie peut être réparti en éléments inconsistants ou sans importance, en éléments qui éclairent le type et la voie, ou qui restent nécessairement dans l'ombre». Bruno Karsenti (2000) a montré que de Comte à Lévi-Strauss, l'homme cesse d'être l'objet d'une représentation consciente pour devenir l'horizon d'une enquête incessamment relancée par l'absence de son objet. 
Claude Lévi-Strauss de projeter sur les mythes amérindiens des opérations binaires qui n'ont de pertinence que dans son propre esprit - informé par la linguistique de Roman Jakobson. Cette objection conduit Sperber à substituer au concept vague $\mathrm{d}^{\prime}$ «esprit humain » chez Lévi-Strauss, conçu comme réserve de significations, un modèle cognitif rigoureux issu des travaux de Noam Chomsky et d'Alan $\mathrm{M}$. Turing ${ }^{9}$. Cependant, Sperber ne fait ainsi que déplacer sur le terrain des sciences cognitives la tentation psychologisante que Lévi-Strauss repérait dans ses propres travaux, et dont il faisait d'abord un rêve littéraire pour en exorciser la hantise.

Il faut en effet revenir à Balzac pour comprendre la formation de l'anthropologie française à travers le projet philosophique, clairement annoncé dans l'avantpropos de La Comédie humaine, de décrire en une seule œuvre l'ensemble des mœurs et des caractères de l'humanité, pensés comme des classifications naturelles dans un esprit d'unité. La quatrième partie de L'Homme nu, intitulée "Scènes de la vie de province ", s'ouvre, au moment où Lévi-Strauss tente de boucler l'immense entreprise des Mythologiques, par cette citation de l'avantpropos de La Comédie humaine:

«La Société ne fait-elle pas de l'Homme, suivant les milieux où son action se déploie, autant d'hommes différents qu'il y a de variétés en zoologie ? Les différences entre un soldat, un ouvrier, un administrateur, un avocat, un oisif, un savant, un homme d'État, un commerçant, un marin, un poète, un pauvre, un prêtre, sont, quoique plus difficiles à saisir, aussi considérables que celles qui distinguent le loup, le lion, l'âne, le corbeau, le requin, le veau marin, la brebis, etc. Il a donc existé, il existera de tout temps des Espèces Sociales comme il y a des Espèces Zoologiques » (Balzac 1940-1950 : I, 4, cité par Lévi-Strauss 1971 : 221).

Cette conception de la Société, que Balzac invente sur le modèle de la zoologie de Geoffroy Saint-Hilaire, repose sur l'intuition d'une profonde unité de la Nature et de la Culture : il y a dans toute la création un ensemble de types qui s'organisent d'après les mêmes formes, ou plus précisément, d'après les mêmes lois de différenciation des formes, en sorte que chaque être social doit être décrit dans le milieu naturel où il prend forme. «Le créateur ne s'est servi que d'un seul et même patron pour tous les êtres organisés. L'animal est un principe qui prend sa forme extérieure, ou, pour parler plus exactement, les différences de sa forme, dans les milieux où il est appelé à se développer » (Ibid.). Il y a certes, selon Balzac, une différence entre

9. Voir Dan Sperber (1974: 69) : «J'admets que l'esprit de Lévi-Strauss est un exemplaire recevable de l'esprit humain, et je ne doute pas que si, par son œuvre, il en révélait les mécanismes, la science aurait franchi un pas considérable. Mais, de même que l'observation d'un athlète ne permet pas de comprendre la physiologie musculaire, de même il s'en faut de beaucoup que l'exercice d'une pensée éminente soit à elle-même sa propre explication. Un modèle de l'esprit humain ne trouve pas sa confirmation de ce qu'il procède d'un esprit humain, car, aussi bien, tous les modèles des plus appliqués aux plus fantaisistes, se valent à cet égard». Voir aussi p. 84 : «On peut être tenté de voir dans ces mythologiques la description d'un langage dont chaque société indienne ne connaîtrait que des bribes enfin rassemblées par Lévi-Strauss. Métaphore splendide, que d'aucuns prennent à la lettre : puisque les mythes relèvent de l'esprit humain et forment ce langage que personne ne parle, alors cet esprit humain n'est l'esprit de personne, entité métaphysique, semblable à l'esprit universel hégélien, dont l'inventeur serait lui-même l'incarnation, Hegel et Napoléon à la fois ». 
la vie sociale et la vie naturelle: c'est que la première est davantage soumise au hasard des rencontres ou des désunions. "La femme d'un marchand est parfois digne d'être celle d'un Prince, et souvent celle d'un prince ne vaut pas celle d'un artiste. L'État social a des hasards que ne se permet pas la Société, car il est la Nature plus la Société » (Ibid.). La séparation entre la Nature et la Culture est donc purement contingente, produit d'événements hasardeux, de ruptures arbitraires ou de disputes sans raisons, et toute la violence de l'histoire vient de ces ruptures: «Le défaut d'union entre deux époux, par quelque cause qu'il soit produit, amène d'effroyables malheurs : nous sommes, tôt ou tard, punis de n'avoir pas obéi aux lois sociales » (Balzac 1940-1950 : I, 4, cité par Lévi-Strauss 1971 : 141).

Mais alors il est possible, selon Balzac, de remonter en deçà de cette séparation contingente entre Nature et Culture pour retrouver l'unité nécessaire du projet du Créateur, à travers l'homologie entre les formes qui les organisent. Celui qui peut retrouver cette unité est donc analogue au Créateur lui-même, capable d'assister au mouvement même par lequel se crée la Société à partir des formes naturelles. Ce génie, c'est le romancier, capable, dit Balzac, de "porter une société tout entière dans sa tête $»^{10}$, s'autoproclamant secrétaire de la Société pour en dresser l'inventaire complet ${ }^{11}$. Celui-ci accomplit alors, selon Balzac, une tâche morale nécessaire : retrouver, derrière la prolifération désordonnée des mœurs les plus diverses, l'unité des lois naturelles qui gouvernent les relations entre l'homme et son milieu. L'espace fictionnel du roman devient le lieu où des personnalités fictives peuvent être construites comme types représentatifs ou emblèmes de toute l'humanité: le roman ne contiendra pas tous les hommes, mais tous les types humains, se clôturant ainsi par sa représentativité ${ }^{12}$.

Pour qu'un tel projet soit accompli, il faut que des conditions particulières soient réalisées: il faut que toutes les espèces sociales viennent se refléter en un lieu où leurs rapports deviennent visibles dans un seul tableau taxinomique. Ce lieu, c'est Paris, espace où convergent toutes les créatures de la province - c'est-à-dire du reste du monde - lieu de culture où la nature se recompose sous la forme de la sauvagerie la plus bigarrée. Chaque parisien est « une encyclopédie vivante, un atlas

10. Voir Balzac, Lettre à Mme Hanska du 6 février 1844: "Quatre hommes ont eu une vie immense: Napoléon, Cuvier, $\mathrm{O}^{\prime}$ Connell et je veux être le quatrième. Le premier a vécu du sang de l'Europe, il s'est inoculé des armées! Le second a épousé le globe ! Le troisième s'est incarné un peuple! Moi j'aurai porté une société tout entière dans ma tête! ( (cité in Grange 1990 : 170).

11. Voir Balzac (1940-1950 : I, 5) : « Le hasard est le plus grand romancier du monde: pour être fécond, il n'y a qu'à l'étudier. La Société française allait être l'historien, je ne devais être que le secrétaire. En dressant l'inventaire des vices et des vertus, en rassemblant les principaux faits des passions, en peignant les caractères, en choisissant les événements principaux de la Société, en composant les types par la réunion des traits de plusieurs caractères homogènes, peut-être pouvais-je arriver à écrire l'histoire oubliée par tant d'historiens, celle des mœurs ».

12. Voir Juliette Grange (1990 : 68) : «La Comédie humaine est une sorte d'encyclopédie des types compris comme singularités significatives en ce qu'elle contient potentiellement tous les "types" humains, tous les systèmes de mœurs depuis la Renaissance, si elle a la forme d'un inventaire, son principe n'est pas la clôture. Elle est un magasin de curiosités: celles qui figurent au catalogue sont significatives, d'autres sont dérisoires, le catalogue est ouvert. L'inventaire est cependant en forme de solde, pour mémoire, à la fin de l'histoire ». Juliette Grange (1996) présente par ailleurs la philosophie de Comte comme un système ouvert, dont la clôture n'est qu'asymptotique. 
grotesque sans cesse en marche et qui jamais ne se repose » : « il représente le monde entier $»^{13}$. Balzac porte ainsi le mouvement muséographique de l'anthropologie française: pour qu'une science de l'homme en société soit possible, il faut que toutes les espèces sociales les plus diverses soient visibles en un seul et même lieu où leurs formes se composent selon des rapports qui prennent enfin une dimension scientifique. Il semble alors que Lévi-Strauss soit pris, à la suite du rêve balzacien, dans une sorte de dilemme ou de double bind : pour qu'une anthropologie soit possible, il faut que les conditions institutionnelles soient réunies afin que, pour la première fois, les faits sur les espèces sociales les plus diverses soient réunis et pensés d'un seul regard de façon totale et scientifique; mais pour celui qui voudra réaliser ce projet, reprenant en lui-même, de façon subjective, une tendance à la totalisation qui traverse toute la société et la constitue en objet, l'issue est le risque de la folie plutôt que le bénéfice du génie. Ce double bind n'est tolérable que s'il déplace ses tensions dans le domaine de la fiction : car c'est seulement de façon fictive qu'un catalogue de toutes les espèces naturelles et sociales est concevable, et c'est par cette fiction qu'un tel projet scientifique devient supportable pour un savant ${ }^{14}$.

S'éclaire alors la place de la référence à la philosophie positive de Comte dans La Pensée sauvage. Au chapitre VIII intitulé «Le temps retrouvé», qui précède la discussion avec Sartre, Lévi-Strauss rend hommage à Comte pour avoir été un des premiers à percevoir le caractère total de la pensée sauvage : «ce grand caractère d'unité de méthode et d’homogénéité de doctrine qui constitue l'état pleinement normal de notre intelligence, et qu'elle avait alors spontanément acquis » (Comte 1975: 270, cité par Lévi-Strauss 1962: 262). Ce qu'il reproche à Comte, c'est d'avoir conçu la pensée sauvage comme une "synthèse subjective ", selon la qualification donnée au fétichisme primitif ${ }^{15}$. Pour Comte, en effet, le fétichisme est la première hypothèse qu'a pu produire l'esprit humain, en projetant de façon fictive sur les choses inanimées du sentiment d'activité que l'homme éprouve spontanément en lui, et qui est le seul phénomène qu'il connaisse originairement en l'absence de toute autre observation ${ }^{16}$. Lévi-Strauss (1962 : 264) refuse alors avec netteté une telle hypothèse originaire:

13. Balzac, La Fille aux yeux d'or et Le Petit mercier (cité in Grange $1990: 202$ ).

14. En ce sens, les critiques adressées par Vincent Descombes à l'anthropologie structurale de Lévi-Strauss (1983 : 232) repèrent, à travers la référence à Balzac, un rêve scientifique qui ne peut tenir sa cohérence que par la fiction. Voir Descombes $(1983: 251)$ : «L'idée d'un répertoire des personnages de La Comédie humaine appartient à la fiction balzacienne plutôt qu'à l'érudition critique. Entreprendre de composer ce répertoire, c'est entrer dans le jeu de Balzac. [...] Tout répertoire se donnant pour un dictionnaire des personnages balzaciens est un appareil fictif. [...] Mais s'il était établi que tout répertoire sera fictif - ne pourra remplir la fonction à laquelle il prétend sinon par fiction - il s'ensuivra que la construction de La Comédie humaine est elle-même fictive ». 15. Voir Comte (1858). Sur la notion de subjectivité chez Comte, voir Arnaud (1973 : 329) : la notion d'Humanité mène à «l'impossibilité de se représenter un sujet qui fût tout sujet sans dire “je") »; et Marquet (1985: 504) : «Le mot subjectivité peut recevoir différents sens chez Comte, et cela d'après les différentes manières dont on entendra le mot objectivité. Si objectif signifie extérieur, subjectif signifie intérieur ; si objectif signifie réel, subjectif signifiera imaginaire ; si objectif signifie présent ou actuel, subjectif signifie passé ou posthume ».

16. Voir Macherey (1989 : 19-27) et Fedi (2000 : 112-118). 
«L'erreur de Comte, et de la plupart de ses successeurs, fut de croire que l'homme a pu, avec quelque vraisemblance, peupler la nature de volontés comparables à la sienne, sans prêter à ces désirs certains attributs de cette nature en laquelle il se reconnaissait ; car s'il avait débuté par le seul sentiment de son impuissance, celui-ci ne lui aurait jamais fourni un principe d'explication. »

Cette objection au positivisme de Comte, pour peu qu'on la décrypte, donne le sens de la référence à Balzac, et l'orientation de ce que l'on peut appeler la prudence méthodologique et éthique de Lévi-Strauss. Le positivisme de Comte est en effet le pendant scientifique du projet littéraire de Balzac, puisqu'il vise à constituer une science totale de la société, sur le modèle des sciences de la nature, en partant du fait de l'organisation commune à tous les êtres sociaux et naturels, et, grâce à cette science, à réorganiser la société en établissant son développement historique sur des bases solides après le désordre de la Révolution française, qui a fait perdre de vue l'existence de lois naturelles de la société. Mais un tel projet de réunification de la Société et de la Nature n'est possible que parce qu'à l'origine, dans les sociétés primitives ou sauvages, nature et culture ne sont pas séparées mais au contraire profondément unies dans une pensée totale où subjectivité et objectivité coïncident. Il faut donc que le savant se fasse porte-parole des Sauvages, ou plutôt apprenne à penser comme un Sauvage, pour retrouver dans la société moderne cette unité de la Nature et de la Culture que les hasards de l'histoire lui ont fait perdre. Le savant positiviste doit donc redevenir " fétichiste » pour boucler dans sa propre subjectivité toute l'histoire de l'humanité ${ }^{17}$.

L'orientation religieuse de la philosophie positive de Comte s'éclaire en effet à partir du thème de plus en plus prégnant d'une "affinité entre le fétichisme et le positivisme ${ }^{18}$. Il est remarquable que ce thème soit lié à une expérience que

17. Ce projet commun à Comte et Balzac est d'abord formulé dans la philosophie politique de Bonald, qu'ils ont lu tous deux attentivement, à travers une analyse de la Révolution française comme événement providentiel rendant visibles tous les états de la pensée sociale, structurée comme un langage, dans le corps sacrifié de la France : "Aujourd'hui que nous avons vu la nation la plus forte et la plus éclairée du globe tomber, dans sa constitution politique, de l'unité de pouvoir la plus concentrée dans la démagogie la plus effrénée et la plus abjecte, et, dans sa constitution religieuse, du théisme le plus parfait à l'idolâtrie la plus infâme ; aujourd'hui que nous avons vu cette même nation revenir, dans son état politique, de cette étonnante dissipation du pouvoir à un usage plus sobre et mieux réglé de l'autorité, et, dans son état religieux, passer de l'absence de tout culte au respect et bientôt à la pratique de son ancienne religion, tous les accidents de la société sont connus, le tour du monde social est fait; nous avons voyagé sous les deux pôles; il ne reste plus de terres à découvrir, et le moment est venu d'offrir la carte de l'univers moral et la théorie de la société » (Bonald 1829 : I, 127-128; je souligne). Bonald est sans doute l'un des premiers à parler de "société primitive» en France, dans le cadre d'une polémique avec la psychologie des Idéologues: «Sans doute ces grandes vérités sont plus sensibles à mesure que l'on remonte aux premiers jours de la société ; car à proprement parler il n'y en a jamais qu'une, et tous les peuples venus, ainsi que tous les hommes, les uns des autres, et toujours au sein de la société, ont retenu, dans leurs transformations successives, la tradition des notions primitives qu'ils avaient reçues et des premiers sentiments dont ils avaient été imbus» (Bonald 1838 : 124 ; je souligne).

18. Voir Comte (1851-1854: III, 119-120) : «Mieux on compare le fétichisme et le positivisme, plus on reconnaît leur affinité fondamentale. Quoique ces deux synthèses extrêmes soient l'une spontanée et l'autre systématique, elles offrent une équivalente subjectivité, condition nécessaire de toute liaison universelle». 
Comte considère comme fondatrice du système positiviste, au sens où elle donne la confirmation de son principe directeur: l'expérience de la crise de folie de 1826 , qui a conduit à un long internement à l'hôpital psychiatrique d'Esquirol ${ }^{19}$. Comte affirme que cette crise lui a permis de passer du positivisme au fétichisme puis de revenir du fétichisme au positivisme, ce qui a vérifié à ses yeux la loi des trois états selon laquelle l'esprit humain passe nécessairement de l'état théologique à l'état positif. Cette expérience a fait l'objet de quelques allusions dans la $52^{\mathrm{e}}$ leçon du Cours de philosophie positive consacrée au fétichisme, puis elle est racontée dans une «Préface personnelle» du cinquième volume du Cours en 1842, pour expliquer le retard pris par la publication des dernières leçons, et enfin elle est pleinement assumée dans le Système de politique positive en 1854 :

«Je me borne seulement à consigner ici la précieuse observation, déjà citée dans nos cours publics, sur ma propre maladie cérébrale de 1826, mentionnée par la préface finale de mon ouvrage fondamental. Une empirique médication ayant prolongé ce trouble pendant huit mois, il en résulta la possibilité de mieux apprécier mes divers états. Or, l'ensemble de cette oscillation exceptionnelle me fit doublement vérifier ma récente découverte envers la principale loi de l'évolution humaine, dont je parcourus alors toutes les phases essentielles, d'abord en sens inverse, puis en sens direct, sans que leur ordre changeât jamais. Le trimestre où l'influence médicale développa la maladie me fit graduellement descendre du positivisme jusqu'au fétichisme, en m'arrêtant d'abord au monothéisme, puis davantage au polythéisme. Dans les cinq mois suivants, à mesure que, malgré les remèdes, ma spontanéité ramena l'existence normale, je remontai lentement du fétichisme au polythéisme, et de celui-ci au monothéisme, d'où je revins promptement à ma positivité préalable. En me procurant aussitôt une confirmation décisive de ma loi des trois états, et me faisant mieux sentir la relativité nécessaire de toutes nos conceptions, ce terrible épisode me permit ensuite de m'identifier davantage avec l'une quelconque des phases humaines, d'après ma propre expérience. »

L'affirmation est étonnante: c'est parce que Comte a fait l'expérience du fétichisme au cours de sa crise de folie qu'il a pu vérifier l'unité de la pensée humaine à travers tous ses états historiques, et les répartir sur une échelle graduée de développement ${ }^{20}$; c'est donc cette expérience singulière qui lui donne l'autorité - on peut bien dès lors parler d'«autorité ethnographique » (Clifford 1988) - pour devenir fondateur de la sociologie et Grand Prêtre de l'Humanité, puisque l'Humanité s'est pensée dans son esprit comme une seule totalité. La cartographie des espèces sociales qu'est la sociologie est possible parce qu'elle s'est réalisée, quoique de façon trop rapide, dans l'esprit d'un seul homme, en sorte qu'il suffit de déplier dans le temps du système ce que Comte a vu en une seule fois par une expérience fulgurante ${ }^{21}$.

19. Sur cette crise de folie et sa signification dans le système positiviste, voir Dumas (1905), ArbousseBastide (1972), Kofman (1978), Descombes (1979), Gouhier (1997), Pickering (1993, 1998).

20. Cette affirmation est d'autant plus étonnante si l'on se rappelle que le positivisme exclut les expériences subjectives au profit de la seule observation des lois objectives des phénomènes. « L'esprit humain peut observer directement tous les phénomènes, excepté les siens propres. [...] L'observation intérieure engendre presque autant d'opinions divergentes qu'il n'y a d'individus croyant s'y livrer » (Comte $1998:$ 33-34).

21. Voir Comte (1975: 250) : «C'est uniquement au très petit nombre d'esprits pleinement philosophiques qui ont pu essentiellement accomplir déjà la grande évolution mentale qu'il .../... 
Comte entreprend donc par la sociologie ce que Balzac entreprend par le roman : penser en une seule fois l'ensemble des espèces sociales sur le modèle de la taxinomie des êtres de la nature. Ces deux tentatives, scientifique et littéraire, forment comme les deux pendants de la reprise d'un thème médiéval : celui de La Divine Comédie de Dante, récit de toutes les singularités humaines les plus communes et les plus ordinaires sous le regard de Dieu. Erich Auerbach, dans Mimesis, a montré que le réalisme moderne trouvait sa source dans la formation par Dante d'un style d'écriture singulier, le "style bas » appliqué aux réalités les plus quotidiennes de la vie sociale, non pour en rire, selon la définition aristotélicienne de la comédie, mais pour les magnifier en les situant dans l'horizon d'un événement final qui les accomplit sous le regard de Dieu, inventant ainsi les figures de ce qu'Auerbach appelle un «réalisme sérieux » ${ }^{22}$. Le projet balzacien de la «comédie humaine » reprend cette inspiration théologique en l'inscrivant dans l'horizon laïcisé d'un roman de l'humanité, où la prise de conscience de la totalité des singularités sociales par un seul homme remplace la hiérarchie divine de l'échelle des êtres telle que Dante la reprenait à la scolastique médiévale ${ }^{23}$. Comte s'inscrit lui aussi dans la postérité de Dante, puisqu'il réclame la rédaction d'une «épopée de l'humanité » qui, s'inspirant de son expérience de la folie, raconterait le passage du fétichisme au positivisme du fondateur de la sociologie - nouveau Dante visitant Les Enfers et revenant d'entre les morts pour en raconter les éclats:

«Idéalisant la philosophie de l'histoire, le poète de l'Humanité caractérisera successivement toutes les phases de la vie préparatoire prolongée jusqu'à l'avènement de l'état final. L'intuition esthétique d'une telle épopée repose sur la crise cérébrale que j'ai décrite à la fin du chapitre un du volume deux, pendant laquelle, après avoir rapidement descendu l'échelle sociologique, je la remontai lentement. Ainsi s'accomplit, suivant tous les âges, une double course, équivalente à l'excursion simple de Dante parmi les divers milieux » (Comte 1851-1854 : IV, 483).

Comment alors situer l'entreprise de Lévi-Strauss, dans ce double horizon scientifique et littéraire - et finalement «théologique » - constitué par l'héritage de Balzac et de Comte ${ }^{24}$ ? Revenons à l'objection qu'il adresse à Comte : son erreur est d'avoir conçu la pensée sauvage comme fétichisme, c'est-à-dire comme projection sur les choses des volontés humaines ; autrement dit, d'être parti de la

[suite de la note 21] appartient aujourd'hui d'entreprendre avec succès de telles comparaisons, à cause de l'heureuse faculté que leur procure exclusivement une entière émancipation personnelle, de transporter presque indifféremment leurs pensées à tous les degrés de l'échelle théologique, sans aucune prédilection perturbatrice ».

22. Voir Auerbach (1968: 203) : «Dante ne voit pas seulement les événements comme un processus humain, comme une histoire terrestre, mais en relation constante avec le plan de Dieu, à l'accomplissement duquel ils concourent sans cesse ».

23. Voir Ibid. : 476 : «Cette vie multiple, saturée d'histoires, crûment représentée dans ses aspects quotidiens, pratiques, triviaux et laids, Balzac [...] la prend au sérieux, et même au tragique, sous cette forme réelle-quotidienne-historique. Depuis que s'était imposé le goût classique, une pareille attitude ne s'était rencontrée nulle part ».

24. Lévi-Strauss utilise parfois la métaphore du retour d'entre les morts, comme dans la polémique avec Roger Caillois; voir Debaene (2004). 
volonté "pure » pour engendrer à partir d'elle l'ensemble des états historiques. C'est oublier, selon Lévi-Strauss, que la volonté humaine n'est jamais pure mais toujours mêlée de façon inextricable aux choses de la nature : l'esprit humain ne pourrait pas se penser lui-même s'il n'y avait déjà dans la nature des choses qui lui font signe. L'erreur de Comte est donc d'avoir conçu le fétichisme comme une "synthèse subjective ", c'est-à-dire comme unification des choses par le sujet, supposant ainsi quelque chose comme une puissance subjective d'unification. Ce que Lévi-Strauss repère chez Comte, c'est donc cette " philosophie du sujet » qu'il retrouvera ensuite chez «la plupart de ses successeurs », Bergson et Sartre au premier chef : la tentation d'unifier de façon primitive des phénomènes qui se donnent d'abord à voir comme des fragments dispersés d'expérience - comme si l'unité ne pouvait être cherchée par le savant sans être d'abord posée par le philosophe à l'origine du savoir. Et ainsi le sujet philosophique remplace Dieu dans l'ordre de la fondation du savoir, dans un horizon qui reste théologique: «leur moi compte davantage que leur dieu » (Lévi-Strauss 1971 : 615).

Comte et Balzac avaient vu cette impossibilité d'une unification originaire des phénomènes, et c'est toute la tension propre au romantisme que d'avoir cherché cette unité, notamment par l'affirmation du sentiment comme puissance spontanée d'unification, sans parvenir à la réaliser par l'intellect. C'est pourquoi Comte - et à sa suite toute la sociologie française, notamment Durkheim et Lévy-Bruhl - a défini l'état primitif de la pensée comme une logique du sentiment, ouvrant ainsi l'aporie dans laquelle tout cette sociologie s'est débattue: comment retrouver dans le discours intellectuel de la science cette unité primitive du sentiment ? Comte a reconnu lui-même qu'une telle expérience conduirait à la folie: "Cet ascendant théorique du sentiment constituerait maintenant un excès de subjectivité, très voisin de la folie $»^{25}$. Définissant la folie comme un "excès de subjectivité ", Comte rend en même temps impossible la clôture du système positiviste : il faut qu'un sujet - le sociologue - pense l'ensemble des états humains de l'origine à la fin de l'histoire, mais cela aboutit à faire l'expérience de la folie, envahissement de l'intellect par une puissance affective venue du «derrière de la tête » et qu'il ne contrôle $\operatorname{pas}^{26}$. La folie comtienne indique donc, audelà de son caractère anecdotique, un problème du discours anthropologique avec le sujet du savoir - sujet qui ne peut connaître son objet qu'en le totalisant et en s'abolissant dans le même mouvement.

Que Lévi-Strauss ait clairement identifié l'aporie d'une telle démarche, c'est ce qu'atteste le statut très particulier de Tristes Tropiques dans son œuvre. Après l'échec de l'élargissement de la méthode structurale aux structures complexes de parenté, qui devait totaliser par la voie purement objective tous les faits

25. Comte (1851-1854 : II, 94). Il faut donc préciser que le fétichisme n'est pas en lui-même fou, mais c'est le fait de penser comme un fétichiste dans une société positiviste qui l'est.

26. Voir Comte (1975 : 250) : «Malgré leur plus grande culture intellectuelle, les hommes qui, pour ainsi, dire, pensent naturellement par le derrière de la tête [...] ont besoin d'exercer presque incessamment sur leurs propres pensées une très active surveillance, pour ne pas se laisser entraîner, dans l'état très prononcé de crainte ou d'espérance déterminé par une passion quelconque, à une sorte de rechute aiguë vers le fétichisme fondamental ». 
humains, Lévi-Strauss recourt à la fiction littéraire pour retrouver le sens de sa démarche anthropologique ; mais c'est précisément pour refuser une expérience originaire qui donnerait d'emblée la totalité du sens du voyage, en explorant, par la méthode du collage de fragments de textes issus de lieux et de moments hétéroclites, ce qu'il appellera ensuite la «logique du sensible » ${ }^{27}$. Et c'est au moment où il s'approche le plus d'une expérience originaire, chez les Nambikwara, qu'il rencontre son double sous la figure d'un chef indien imitant son écriture, scène qui suscite en lui un tel tourment qu'il lui faut l'apaiser par une philosophie de l'histoire de l'écriture ${ }^{28}$.

Cette identification de l'aporie du sujet anthropologique éclaire le passage de Rousseau à Montaigne dans les références de Lévi-Strauss (Désveaux 1992). Si Rousseau est celui qui a tenté de réconcilier dans sa subjectivité le moi et l'autre, au prix d'une douloureuse schizophrénie (Lévi-Strauss 1996 : 48-49), Montaigne apparaît dans les derniers textes de Lévi-Strauss comme le témoin d'un événement majeur qui sépare définitivement l'humanité d'avec elle-même et rend impossible sa réconciliation, même en pensée: l'événement de la Conquête, à travers lequel une partie de l'humanité constitue l'autre moitié en objet. "Ce repli sur soi, cette frilosité, cette cécité volontaire furent la première réplique d'une humanité qui se croyait pleine et entière, mise du jour au lendemain devant cette évidence: du genre humain elle ne formait qu'une moitié » (LéviStrauss 1991 : 291). La référence finale à Montaigne n'est donc pas le constat désabusé d'un relativisme culturel devant la diversité des coutumes humaines; c'est la solution provisoire trouvée par Lévi-Strauss à un problème qui n'a cessé de l'inquiéter, comme il a inquiété tout le XIX ${ }^{\mathrm{e}}$ siècle: comment unifier par la pensée une humanité séparée du reste de la nature et d'elle-même? La position de Montaigne est celle d'un témoin : témoin de la folie moderne qui se refuse à devenir fou lui-même pour guérir cette folie, et qui tente simplement d'introduire dans sa propre subjectivité ce dédoublement par lequel l'humanité s'est séparée de la nature et d'elle-même. " "Nous n'avons aucune communication à l'être" : tout se résume dans ces paroles décisives qu'on ne se lasse pas de citer. Et convaincus de ce manque, nous ne savons même plus si ce savoir qui se nie luimême en est un » (Ibid. : 286). Il ne s'agit plus alors de faire communiquer toutes les formes de la pensée humaine mais de constater que «nous n'avons aucune communication à l'être ", et d'en tirer une sagesse, au double sens d'une règle de vie et d'un type de savoir. Ne pas partir de la puissance d'un sujet pour unifier l'ensemble des phénomènes, mais partir du sentiment subjectif d'impuissance pour en tirer une forme de savoir et une règle de vie.

27. Lévi-Strauss (1955: 44) : «Des événements sans rapport apparent, provenant de périodes et de régions hétéroclites, glissent les unes sur les autres et soudain s'immobilisent en un semblant de castel dont un architecte plus sage que mon histoire eût médité les plans.[... I Il a fallu vingt années d'oubli pour m'amener au tête-à-tête avec une expérience ancienne dont une poursuite aussi longue que la terre m'avait jadis refusé le sens et ravi l'intimité». Sur la méthode d'écriture de Tristes Tropiques, voir Debaene (2002).

28. Lévi-Strauss (1955 : 350-355). Jacques Derrida (1967), habile à traquer les figures de folie qui déconstruisent de l'intérieur des systèmes, a concentré sur ce point sa lecture. 
Si Montaigne remplace Rousseau dans les derniers textes de Lévi-Strauss, le modèle de l'opéra wagnérien remplace celui de La Comédie humaine. On dira qu'entre Balzac et Wagner il y a la même ambition de totalisation historique caractéristique du XIX siècle. Mais d'une part, La Comédie humaine repose sur l'expérience de la vision, celle du tableau taxinomique qui embrasse d'un seul coup d'œil l'ensemble des classifications naturelles et sociales, alors que l'opéra wagnérien repose sur l'expérience de l'écoute, inscrite dans une temporalité faite d'attentes et de surprises, de chutes et de plénitudes, à travers un parcours sur lequel toute saisie totalisatrice est impossible (Lévi-Strauss 1964 : 24-24 ; 1971 : 588-589). D'autre part, alors que La Comédie humaine cherche à rendre visible les moindres détails de la vie quotidienne par le regard d'un seul homme, selon une ambition démesurée que Comte appelle " excès de subjectivité », l'opéra wagnérien prend pour point de départ un défaut de communication, et tente de trouver une «bonne distance » entre le défaut et l'excès de communication. Dans un article intitulé "De Chrétien de Troyes à Richard Wagner», Lévi-Strauss (1983) semble prendre le contre-pied de la généalogie du réalisme établie par Auerbach, en traçant un lien non pas entre Dante et Balzac, La Divine Comédie et La Comédie humaine, mais entre Chrétien de Troyes et Wagner, à travers le motif de la quête du Graal par Perceval le Gallois. Comme dans La Divine Comédie, Perceval passe du monde naturel des vivants au monde surnaturel des morts ; mais au lieu de voir la vie totale des hommes dans le moment de leur mort, il entend la réponse à une question qu'il n'a pas posée ; et au lieu de recevoir cette réponse comme une vision totale unissant l'intellect et la sensibilité, il l'accueille de façon nécessairement partielle dans un élan affectif de pitié. Wagner se trouve ainsi rejoindre Rousseau, penseur de «l'identification à autrui [comme] mode originel de communication, antérieur à l'émergence de la vie sociale et du langage articulé, capable d'unir les hommes entre eux et avec toutes les formes de vie ", et Montaigne, "penseur du "connaître et ne pas connaître", c'est-à-dire savoir ce qu'on ignore, "Durch Mitleid wissend": non par un acte de communication mais par un élan de pitié, qui fournit une issue au dilemme dans lequel son intellectualisme, longtemps méconnu, risquait d'emprisonner la pensée mythique » (Lévi-Strauss 1983 : 315 et 318). Et ainsi Lévi-Strauss, à défaut de pouvoir écrire un roman ou une pièce de théâtre qui synthétise l'ensemble de sa pensée - nouvelle «Comédie humaine » - parvient à écrire l'équivalent d'un opéra.

Il faudrait donc distinguer deux modes opératoires de la fiction dans l'œuvre de Lévi-Strauss et dans le discours anthropologique en général : une fiction picturale, qui donne la saisie complète de son objet dans l'évidence d'un tableau taxinomique, faisant ainsi coïncider le sujet et l'objet de la connaissance, dans l'ambivalence du génie (si l'on se place du point de vue du Créateur infini) et de la folie (si l'on se place du point de vue de la créature finie) ; et une fiction musicale, qui fait participer le sujet à la fabrication interne d'un objet en train de se faire, dans le jeu d'opérations formelles éprouvées subjectivement comme des 
qualités sensibles. L'alternative entre la science et la fiction n'en est une que dans le premier mode de fictionnalisation, car elle ouvre à la possibilité de recourir à la fiction dans l'échec de la science, selon une opposition classique entre la sécheresse de l'intelligence et la richesse de l'émotion ; mais elle ne tient plus dans le second modèle, car alors la fiction est une procédure nécessaire dans la connaissance d'un objet en cours de fabrication, dont les transformations impliquent de façon immanente une compétence fictionnelle et une opération cognitive, une altération affective et une saisie conceptuelle. L'inquiétude que le fétichisme a introduite dans le positivisme éclate ainsi au grand jour : il n'y a de connaissance positive des faits que dans la mise en œuvre d'une compétence fictionnelle, que l'on peut bien qualifier alors de «primitive » ou de «sauvage».

MOTS CLÉS/KEYWORDS: fétichisme/fetishism - sujet/subject - classification - totalisation expérience picturale/pictorial experience - expérience musicale/musical experience - Honoré de Balzac - Claude Lévi-Strauss - Auguste Comte.

\section{BIBLIO GRAPHIE}

\section{Arbousse-Bastide, Paul}

1972 "Auguste Comte et la folie», in Roger Bastide, ed., Les Sciences de la folie. Paris, PUF : 47-72.

\section{Arnaud, Pierre}

1973 Le Nouveau Dieu. Paris, Vrin.

\section{Auerbach, Erich}

1968 Mimesis. La représentation de la réalité dans la littérature occidentale. Paris,

Gallimard.

\section{Balzac, Honoré de}

1940-1950 La Comédie humaine. Paris, Gallimard («Bibliothèque de la Pléiade»).

\section{Bonald, Louis de}

1829 Législation primitive: œưres complètes. Paris, Adrien Leclerc \& $C^{\mathrm{ie}}, 3$ vol.

1838 Recherches philosophiques sur les premiers objets des connaissances morales: cuvres complètes, IX. Paris, Adrien Le Clerc \& $C^{i e}$.

\section{Canguilhem, Georges}

1994 «Histoire des religions et histoire des sciences dans la théorie du fétichisme chez Auguste Comte ", in Études d'histoire et de philosophie des sciences. Paris, Vrin : 81-98.

\section{Clifford, ames}

1988 «On Ethnographic Authority», in The Predicament of Culture, TwentiethCentury Ethnography, Literature and Art. Cambridge, Harvard University Press : 2154 [Trad. fr. : Malaise dans la culture. Paris, Éd. de l'École nationale des Beaux-Arts, 1996].

\section{Comte, Auguste}

1851-1854 Système de politique positive. Paris, Corillian-Gœury \& Dalmont.

1858 Synthèse subjective. Paris, Dalmont. 1975 [1830-1842] Cours de philosophie positive, «Leçons 46 à 60 ». Paris, Hermann. 1998 [1830-1842] Cours de philosophie positive, «Leçons 1 à 45 ». Paris, Hermann.

\section{Debaene, Vincent}

2002 «L'adieu au voyage. À propos de Tristes Tropiques», Gradhiva 32 : 13-36.

2004 «Portait de l'ethnologue en Lazare» in Michel Izard, ed., Cahiers de L'Herne: Lévi-Strauss: 99-107.

\section{Delvolvé, tean}

1932 Réflexions sur la pensée comtienne. Paris, Alcan. 


\section{Derrida, tacques}

1967 De la grammatologie. Paris, Éd. de Minuit.

\section{D escombes, Vincent}

1978 "L'utopie positive", in Régénération et reconstruction sociale entre 1780 et 1848 .

Paris, Vrin.

1979 «À propos des crises cérébrales d'Auguste Comte», Revue philosophique 1 : 67-81.

1983 «Who's who dans La Comédie humaine de Balzac ", in Grammaire d'objets en tous genres. Paris, Éd. de Minuit : 251-280.

\section{Désveaux, Emmanuel}

1992 «Un itinéraire de Lévi-Strauss. De Rousseau à Montaigne», Critique 540 : 374-390.

\section{D umas, Georges}

1905 Psychologie de deux messies positivistes: Saint-Simon et Auguste Comte. Paris, Alcan.

\section{Fedi, Laurent}

2000 Comte. Paris, les Belles Lettres.

\section{Gouhier, Henri}

1987 La Philosophie d'Auguste Comte. Paris, Vrin.

1997 [1931] La Vie d'Auguste Comte. Paris, Vrin.

\section{Grange, dliette}

1990 Balzac, l'argent, la prose, les anges. Paris, La Différence.

1996 La Philosophie d'Auguste Comte. Paris, PUF.

\section{lacono, Alfonso}

1992 Le Fétichisme: histoire d'un concept. Paris, PUF.

\section{Karsenti, Bruno}

1997 L'Homme total : sociologie, anthropologie et philosophie chez Marcel Mauss. Paris, PUF.
2000 «Le problème des sciences humaines. Comte, Durkheim, Lévi-Strauss ", Archives de philosophie 63 : 445-465.

\section{Keck, Frédéric}

2004 Lévi-Strauss et la pensée sauvage. Paris, PUF.

\section{Kofman, Sarah}

1978 Aberrations: le devenir-femme

d'Auguste Comte. Paris, Aubier-Flammarion.

\section{Latour, Bruno}

1996 Petite réflexion sur le culte moderne des dieux faitiches. Paris, Synthélabo.

\section{Lepenies, W olf}

1990 «Les métamorphoses d'Auguste Comte", in Les Trois Cultures: entre science et littérature, l'avènement de la sociologie. Paris, Éd. de la Maison des sciences de l'homme.

\section{Lévi-Strauss, Claude}

1955 Tristes Tropiques. Paris, Plon.

1962 La Pensée sauvage. Paris, Plon.

1964 Le Cru et le Cuit. Mythologiques I.

Paris, Plon.

1968 L'Origine des manières de table. Mythologiques III. Paris, Plon.

1971 L'Homme nu. Mythologiques IV. Paris, Plon.

1983 «De Chrétien de Troyes à Richard Wagner ", in Le Regard éloigné. Paris, Plon.

1991 Histoire de Lynx. Paris, Plon.

1996 [1973] «Jean-Jacques Rousseau fondateur des sciences de l'homme » in Anthropologie structurale deux. Paris, Plon.

\section{Lévy-Bruhl, Lucien}

1900 La Philosophie d'Auguste Comte. Paris, Alcan.

\section{Littré, Émile}

1862 Auguste Comte et la philosophie positive. Paris, Hachette. 


\section{Lukács, Georg}

218

1967 Balzac et le réalisme français. Paris, Maspéro.

\section{Macherey, Pierre}

1989 Comte, la philosophie et les sciences.

Paris, PUF.

\section{Marquet, tean-François}

1985 «Religion et vie subjective chez

Comte», Revue philosophique : 501-517.

\section{Pickering, Mary}

1993 Auguste Comte: An Intellectual

Biography. Cambridge, Cambridge

University Press.
1998 «Auguste Comte and the Return to

Primitivism ", Reoue internationale de

philosophie 203 : 51-77.

\section{Pouillon, tean}

1975 Fétiches sans fétichisme. Paris, Maspéro.

\section{Sartre, tean-Paul}

1960 Critique de la raison dialectique. Paris, Gallimard.

\section{Schaeffer, tean-Marie}

1999 Pourquoi la fiction? Paris, Le Seuil.

\section{Sperber, D an}

1974 Le Symbolisme en général. Paris, Hermann.

RÉSU MÉ/ ABSTRACT

Frédéric Keck, Fiction, folie, fétichisme : Claude Lévi-Strauss entre Comte et La Comédie humaine. - La Pensée sauvage de Claude Lévi-Strauss se construit sur une double référence à La Comédie humaine de Balzac et à l'analyse du fétichisme chez Auguste Comte. Ce double régime d'écriture, littéraire et scientifique, résout la tension entre un projet objectif de totalisation des données ethnographiques sur les sociétés humaines les plus diverses et sa reprise dans l'expérience d'un sujet - tension qui était déjà au cœur de l'analyse du fétichisme chez Comte, désignant à la fois un état passé de l'humanité et l'expérience de la folie pour le sujet sociologue. On conclut que cette tension est dénouée par Claude Lévi-Strauss dans le passage d'un modèle pictural de la fiction - celui du tableau des classifications - à un modèle musical - celui de l'expérience d'une écoute.
Frédéric Keck, Fiction, Insanity, Fetishism :Claude Lévi-Strauss between Comte and Balzac. -

Claude Lévi-Strauss's The Savage Mind is built on references to both Honoré de Balzac's The Human Comedy and Auguste Comte's analysis of fetishism. This double, literary and scientific, writing settles the tension between the objective of totalizing ethnographic data about the most diverse human societies and the use of this in a subject's experience. This tension already laid at the core of Comte's analysis of fetishism, which referred to both a past state of humanity and the experience of insanity. LéviStrauss resolves this tension by switching from a pictorial model of fiction (the table of classifications) to a musical model (the listening experience). 